\title{
TRAIL overexpression co-regulated by Egr1 and HRE enhances radiosensitivity of hypoxic A549 cells depending on its apoptosis inducing role
}

\author{
YAN-MING YANG ${ }^{1 *}$, FANG FANG ${ }^{2,3}, \mathrm{XIN} \mathrm{LI}^{2}$, LEI YU $^{1 *}$ and ZHI-CHENG WANG ${ }^{2 *}$ \\ ${ }^{1}$ Department of Radiotherapy, Second Hospital of Jilin University, Changchun, Jilin 130041; \\ ${ }^{2}$ Key Laboratory of Radiobiology, Ministry of Health, School of Public Health, \\ Jilin University, Changchun, Jilin 130021; ${ }^{3}$ Department of Nutrition and Food Hygiene, \\ School of Public Health, Jilin University, Changchun, Jilin 130021, P.R. China
}

Received June 22, 2016; Accepted November 17, 2016

DOI: 10.3892/or.2016.5271

\begin{abstract}
Ionizing radiation can upregulate the expression levels of TRAIL and enhance tumor cell apoptosis. While Early growth response 1 (Egr1) gene promoter has radiation inducible characteristics, the expression for exogenous gene controlled by Egrl promoter could be enhanced by ionizing radiation, but its efficiency is limited by tissue hypoxia. Hypoxia response elements (HREs) are important hypoxic response regulatory sequences and sensitivity enhancers. Therefore, we chose TRAIL as the gene radiotherapy to observe whether it is regulated by Egr1 and HER and its effects on A549 cells and its mechanism. The pcDNA3.1-Egr1-TRAIL (pc-E-hsT) and pcDNA3.1-HRE/Egr1-TRAIL (pc-H/E-hsT) plasmids containing Egr1-hsTRAIL and HRE/Egr1-hsTRAIL were transfected into A549 cells, the cells were treated by hypoxia and radiation. The TRAIL mRNA in the cells and protein concentration in the culture supernatants were measured by RT-PCR and ELISA, respectively. Mean lethal dose $\mathrm{D}_{0}$ value was evaluated with colony forming assay. The cell apoptotic rates were analyzed by FCM and TUNEL assay. Expression of DR4, DR5 and cleaved caspase-3 proteins were analyzed by western blotting. It showed that TRAIL mRNA expression and TRAIL concentration all significantly increased under hypoxia
\end{abstract}

Correspondence to: Dr Zhi-Cheng Wang, Key Laboratory of Radiobiology, Ministry of Health, School of Public Health, Jilin University, 1163 Xinmin Street, Changchun, Jilin 130021, P.R. China E-mail: zhicheng123a@126.com

Dr Lei Yu, Department of Radiotherapy, Second Hospital of Jilin University, 218 Ziqiang Street, Changchun, Jilin 130041, P.R. China E-mail: yul0826@163.com

*Contributed equally

Key words: human secreted TRAIL, early growth response 1, hypoxia response elements, radiosensitivity, apoptosis and/or radiation. $\mathrm{D}_{0}$ value of $\mathrm{pc}-\mathrm{H} / \mathrm{E}-\mathrm{hs}$ T transfected cells under hypoxia was lowest, indicating more high radiosensitivity. Hypoxia could not cause the pc-E-hsT transfected cell apoptotic rate increase, but there were promoting effects in pc-H/E-hsT transfected cells. DR4 had not obvious change in pc-E-hsT and pc-H/E-hsT transfected cells under normoxic and hypoxic condition, otherwise, DR 5 and cleaved caspase-3 increased mostly in pc-H/E-hsT transfected cells under hypoxic condition. TRAIL overexpression was co-regulated by Egr1 and HRE. TRAIL might promote hypoxic A549 cell radiosensitivity and induce apoptosis depending on DR5 to caspase-3 pathways.

\section{Introduction}

Lung cancer is the most common malignancy, its mortality ranks first, and more than a million of patients die from lung cancer each year world-wide. Although surgery, radio- and chemotherapy, and other treatments all were used for lung cancer, but the therapeutic results are limited. Therefore, the combined therapy strategy used to kill the tumor cells has become a hotspot in the field of cancer treatment research. Cancer treated by genetic means combined with radiotherapy is a method proposed recently (1). The enhancements of tumorkilling gene expression and radiotherapy produce synergistic effects to inhibit tumors.

With the aim to improve the clinical outcomes of locally advanced cancer, various radiotherapeutic approaches have been implemented. Tumor gene-radiotherapy is a strategy proposed for a large quantity of genes inducing tumor cell apoptosis, inhibiting oncogenic activity, reducing angiogenic activity to sensitize cells to radiation. Usually, tumor cell apoptosis resistances is a central hallmark of carcinogenesis, consequently the efficacy of all anti-tumor treatments is limited by the presence of cells displaying alterations in their apoptotic machinery (2). Selective induction of apoptosis in malignant cells may represent a central therapeutic strategy in radiation oncology (3). Tumor necrosis factor related apoptosis inducing ligand (TRAIL) was coloned from human lymphocytes and cardiac muscle cDNA by Wiley et al (4). In recent 
years, by virtue of its pro-apoptotic effect on cancer cells, but not normal cells, TRAIL has been considered a promising candidate for tumor gene therapy, even tumor gene-radiotherapy (5-8). Gene-radiotherapy based on TRAIL has significant synergistic effect, which has improved curative effect in breast cancer treatment of preclinical experiments and I-II stage clinical trials (9). Some studies confirm that ionizing radiation can upregulate the expression levels of TRAIL and death receptors (DR4 and DR5) and promote the enhancement of tumor cell apoptosis. DR4 and DR5 receptors have pro-apoptotic activity, but decoy receptors DcR1 and DcR2, and osteoprotegerin have none (7). DR4 and DR5 are the only proapoptotic TRAIL-receptors, that may regulate apoptosis by caspase- 8 or caspase -10 to caspase- 3 pathways (10).

Gene expression enhancement mediated radiation inducible promoter became impossible in tumor gene-radiotherapy, the early growth response 1 (Egr1), also known as NGFI-A, zif268, TIS8, cef5 and krox24, has been shown to have the characteristics, it contains 6 serum response elements, $\left[\mathrm{CC}(\mathrm{A} / \mathrm{T})_{6} \mathrm{GG}\right]$, which are essential for Egrl gene activation by radiation (11). In 1992, Weichselbaum and his collegues concluded that Egr1 promoter could be linked to encoding region of exogenous genes to activate the transcription and to enhance protein expression levels by ionizing radiation (12). One by one, many studies have confirmed the radiationinducible characteristics of Egr1 promoter, the exogenous genes included TNF- $\alpha$, IFN- $\gamma$, endostatin and TRAIL, etc, gene-radiotherapy based on Egr1 promoter became a hotspot of radiation oncology $(8,13-15)$. It is well known that hypoxia is a common phenomenon in solid tumor development, it causes tumor radio- and chemotherapy resistance. In addition, Egr1 promoter inducible activity by radiation was greatly reduced under hypoxic condition, which was caused by oxygen-free radical reduction $(16,17)$. Hypoxia response elements (HREs) are important hypoxic response regulatory sequences and sensitivity enhancers, and have higher activity in solid tumor. Some studies constructed HRE and Egr1 dual-sensitive promoter, and gene transcriptional activity was enhanced significantly under hypoxic conditions, which might make hypoxia a contributing factor for radiotherapy treatment $(18,19)$.

In order to achieve the maximum effects of tumor generadiotherapy, in the current study, pro-apoptotic gene human secreted TRAIL (hsTRAIL) was mediated by dual sensitivity promoter HRE/Egr1 to form recombinant expression plasmid pc-H/E-hsT, and pc-E-hsT was used as a control. After transient transfection into human lung adenocarcinoma A549 cells, the radiosensitivity under normoxic and hypoxic condition was evaluated, to determine whether pc-H/E-hsT may serve as a novel gene-radiotherapeutic agent for cancer treatment.

\section{Materials and methods}

Construction of recombinant plasmid. The pshuttle-hsTRAIL and pMD19T-Egr1 plasmids were constructed and kindly given by Dr Yan-bo Li, School of Public Health and Family Medicine, Capital Medical University. Egr1 fragment was obtained from pMD19T-Egrl vector digested by XbaI and HindIII, hsTRAIL fragment was obtained from pshuttle-hsTRAIL vector digested by KpnI and BamHI, HRE dual-strand containing $M l u \mathrm{I}$ and SacI restriction site were by Biochemical synthesis (Sangon Biotech Co., Ltd., Shanghai, China). The pc-E-shT and $\mathrm{pc}-\mathrm{H} / \mathrm{E}-\mathrm{shT}$ plasmids were constructed by gene recombination technique as shown in Fig. 1.

Cell culture and transfection. Human lung adenocarcinoma A549 cells were obtained from American Type Culture Collection (ATCC, Manassas, VA, USA). A549 cells were maintained in Dulbecco's modified Eagle's medium (DMEM) (Invitrogen, Carlsbad, CA, USA). DMEM was supplemented with $10 \%$ fetal bovine serum (FBS) (Invitrogen), penicillin (Invitrogen) $(100 \mathrm{U} / \mathrm{ml})$, streptomycin sulfate (Invitrogen) $(100 \mu \mathrm{g} / \mathrm{ml})$, at $37^{\circ} \mathrm{C}$ in a humidified $5 \% \mathrm{CO}_{2}$ atmosphere. The cells were transfected with the Lipofectamine ${ }^{\mathrm{TM}} 2000$ (Invitrogen) reagent according to the manufacturer's protocol. Cells were incubated for $6 \mathrm{~h}$, then the transfection medium were replaced by fresh complete growth medium.

Cell hypoxia and irradiation. A549 cells were transfected with pc-E-hsT and pc-H/E-hsT as described above. After $24 \mathrm{~h}$, $\mathrm{CoCl}_{2}$ (Sigma, St. Louis, MO, USA) were added into the wells of plate at $150 \mu \mathrm{mol} / 1$ final concentration to study hypoxia, after $24 \mathrm{~h}$, cells were exposed to X-rays using an X-ray generator (model XSZ-Z20/20, Dandong, Liaoning, China) with $200 \mathrm{kV}$ and $18 \mathrm{~mA}$. The dose rate of irradiation was $0.342 \mathrm{~Gy} / \mathrm{min}$, 4 Gy total dose was used (8).

RT-PCR and ELISA. A549 cells were seeded into 6-well plates by $5 \times 10^{5}$ cells/well, after transfection, hypoxia and irradiation as described above, culture supernatant were collected to measure TRAIL concentration by enzyme-linked immunosorbent assay (ELISA). While total RNA were extracted using TRIzol reagent (Invitrogen). RNA of each sample (200 ng) was synthesized into cDNA by RT-PCR kits (Takara, Dalian, China). Following primers were synthesized by Takara, GAPDH forward: 5'-TATTGGGCGCCTGGTCACCA-3', reverse: 5'-CCACCTTCTTGATGTCATCA-3'; amplicons were $187 \mathrm{bp}$; hsTRAIL forward: 5'-CATCTATTCCCAAAC ATACTT-3', reverse: 5'-CCCTTGATAGATGGAATAGA-3', amplicons were $746 \mathrm{bp}$. The PCR reaction were performed: $94^{\circ} \mathrm{C}$ for $5 \mathrm{~min} ; 94^{\circ} \mathrm{C}$ for $30 \mathrm{sec}, 62^{\circ} \mathrm{C}$ for $30 \mathrm{sec}$, and $72^{\circ} \mathrm{C}$ for $45 \mathrm{sec}, 30$ cycles; $72^{\circ} \mathrm{C}$ for $10 \mathrm{~min}$. Amplified products were separated in $1 \%$ agrose gels and visualized using ethidium bromide staining. Band intensities were quantified using a gel imaging instrument and Quantity One software (Bio-Rad Laboratories, Inc., Hercules, CA, USA). The hsTRAIL concentration in the culture supernatants were measured by ELISA kits (R\&D Systems, Inc., Minneapolis, MN, USA) according to the manufacturer's protocol.

Colony forming assay. A549 cells were seeded into $60-\mathrm{mm}$ dish by $5 \times 10^{5} /$ dish, after transfection and hypoxia as described above. Cells were digested, then they were seeded again into $60-\mathrm{mm}$ dish at $1 \times 10^{2} / \mathrm{dish}(0 \mathrm{~Gy}), 2 \times 10^{2} / \mathrm{dish}(2 \mathrm{~Gy})$,

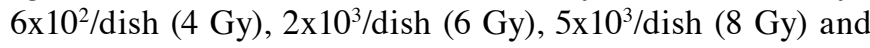
$5 \times 10^{4} /$ dish (10 Gy). The DMEM was replaced every 2 days for 10 days. Cells were fixed with methanol and dyed with Giemsa, the colonies were counted. The survival fraction (SF) 


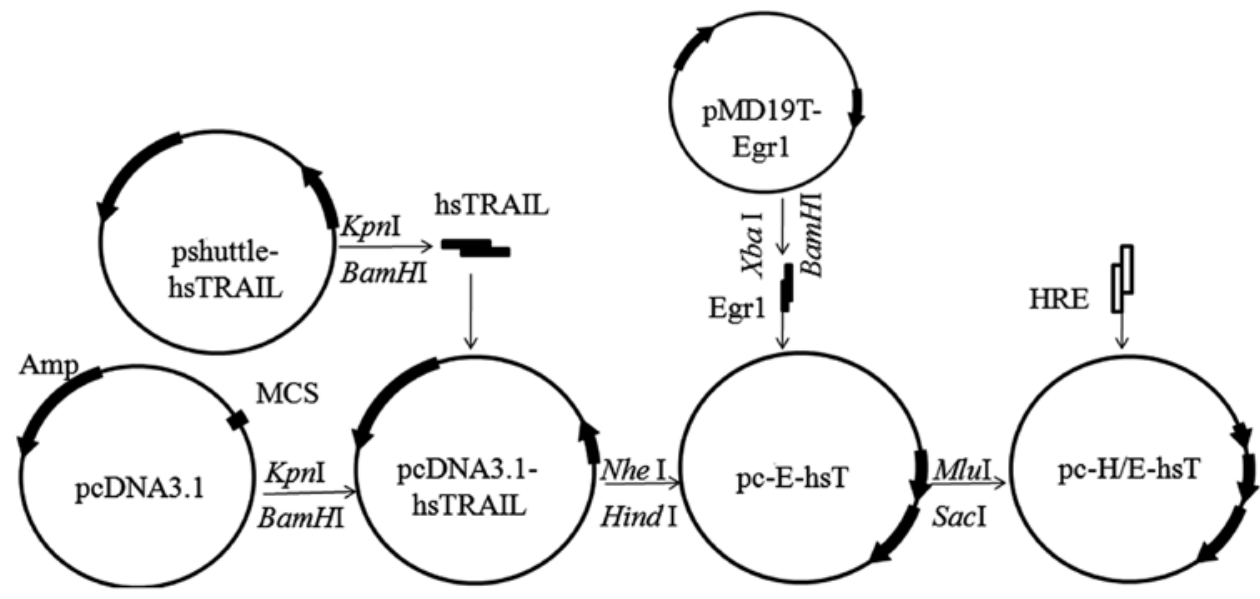

Figure 1. Construction of recombinant plasmid pc-E-hsT and pc-H/E-hsT.
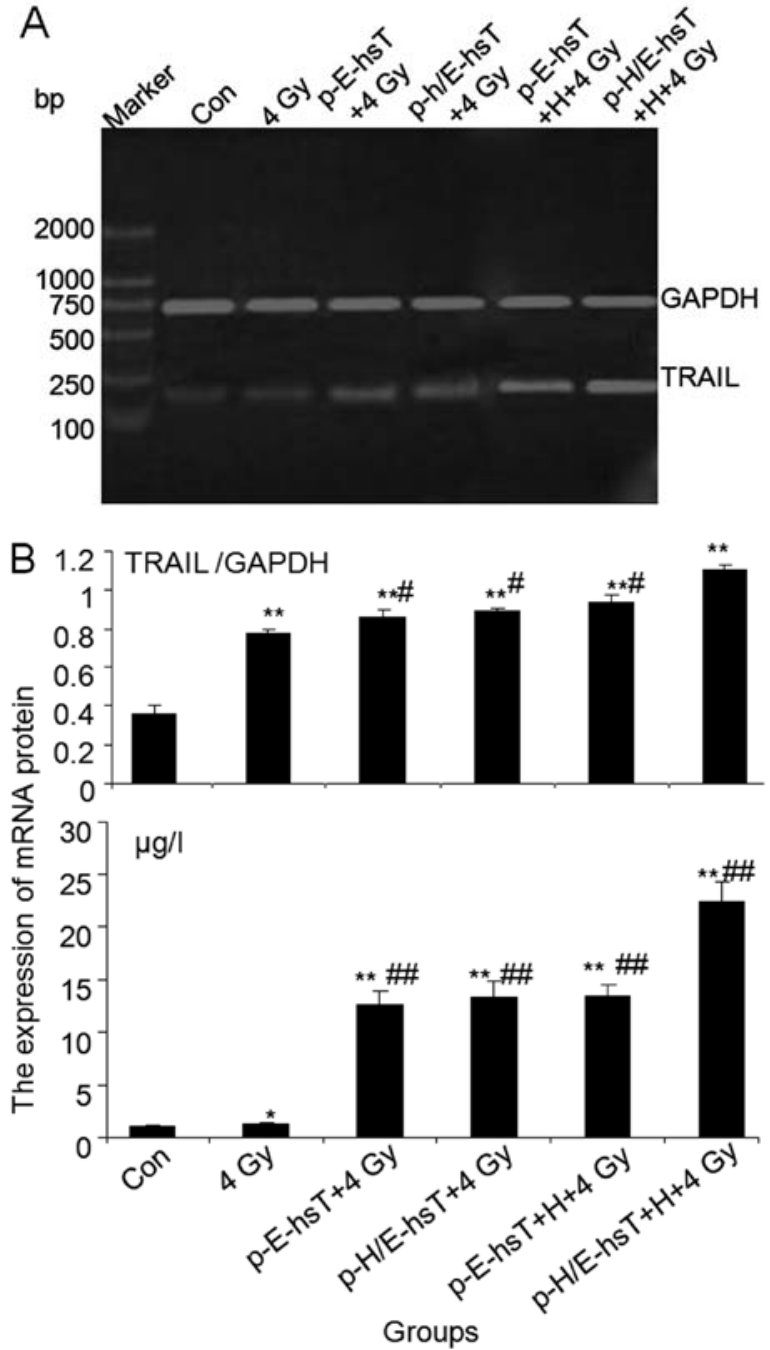

Figure 2. Detection of TRAIL mRNA in cells and protein in culture supernatant by hypoxia $(\mathrm{H})$ and irradiation. (A) TRAIL PCR product electrophoresis, GAPDH was used as an loading control. (B) Upper: Quantification of TRAIL mRNA levels. Lower: Concentration of secreted TRAIL protein by ELISA. ${ }^{*} \mathrm{P}<0.05,{ }^{* *} \mathrm{P}<0.01$ as compared with control group, ${ }^{\#} \mathrm{P}<0.05,{ }^{\# \#} \mathrm{P}<0.01$ as compared with $4 \mathrm{~Gy}$.

was calculated upon the rate of treated group colony number to untreated group colony number. Mean lethal dose $\mathrm{D}_{0}$ value was calculated using linear correlation and regression analysis. A smaller $\mathrm{D}_{0}$ value indicates a higher radiosensitivity.

Flow cytometry. Apoptotic rate was measured by Annexin V staining of externalized phosphatidylserin in apoptotic cells using FCM (Becton-Dickinson Co., Franklin Lakes, NJ, USA) with Annexin V/FITC kit (KeyGen Biotech, Nanjing, China). Briefly, A549 cells were seeded into 24-well plate by $1 \times 10^{5} /$ well, after transfection, hypoxia treatment and irradiation as described above, cells were harvested and washed with phosphate-buffered saline (PBS) 3 times, and stained with Annexin V-FITC and propidium iodide (PI) (KeyGen Biotech) for $10 \mathrm{~min}$ at room temperature according to the protocol of the manufacturer. The apoptotic cells were analyzed by FCM, which display Annexin $\mathrm{V}^{+} / \mathrm{PI}^{-}$(early apoptosis) or Annexin $\mathrm{V}^{+} / \mathrm{PI}^{+}$staining (late apoptosis) (20). There were 3 replicate wells per group. The experiment was performed in triplicate.

TUNEL assay. A549 cells were seeded into 24-well plate with clean coverslip by $5 \times 10^{4} /$ well, after transfection, hypoxia treatment and irradiation as described above, the coverslip were removed and fixed with $4 \%$ paraformaldehyde, TUNEL (terminal deoxynucleotidyl transferase-mediated dUTP-biotin nick end labeling) (KeyGen Biotech) reaction was performed according to the manufacturer's protocol. The cells were stained with diaminobenzidine (DAB) for $10 \mathrm{~min}$ and counterstained with Mayrow hematoxylin. TUNEL positive cells from 100 cells in one visual field under a microscope were counted, 3 fields were randomly selected for one sample.

Western blotting. A549 cells were seeded into 6-well plates at $5 \times 10^{5}$ cells/well, after transfection, hypoxia and irradiation as described above, cells were collected into Eppendorf tubes, total proteins were extracted with cold lysis buffer (10 mmol/1 Tris-HCl, pH 7.4; $1 \mathrm{mmol} / 1$ EDTA, pH 8.0; $0.1 \mathrm{~mol} / 1 \mathrm{NaCl} ; 1 \mu \mathrm{g} / \mathrm{ml}$ aprotinin; $100 \mu \mathrm{g} / \mathrm{ml} \mathrm{PMSF}$ ), and the protein concentration was determined by using Coomassie brilliant blue protein assay from Jiancheng Bioengineering Institute (Nanjing, China). For western blotting, $25 \mu \mathrm{g}$ protein was separated using sodium dodecyl sulfate-polyacrylamide gel electrophoresis (SDS-PAGE), and then transferred to 

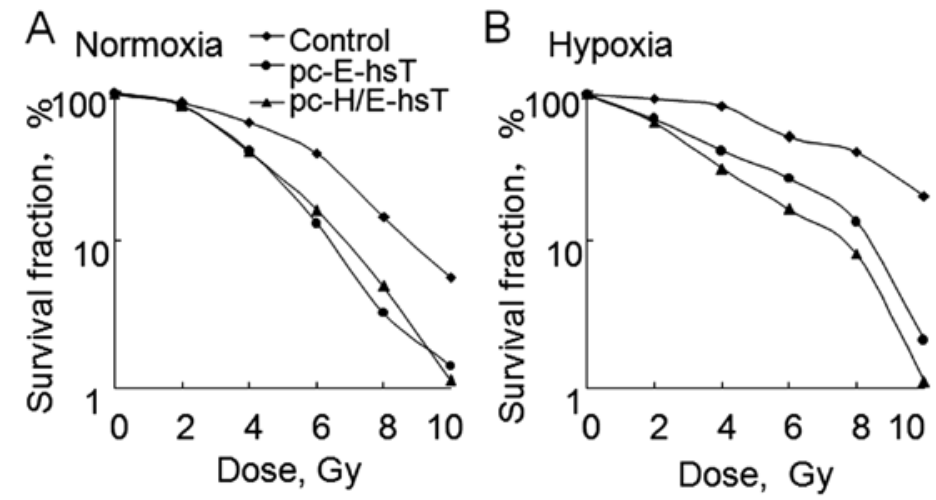

Figure 3. Dose-survival curve of A549 cells under normoxic and hypoxic condition. (A) Under normoxic condition; (B) under hypoxic condition.

nitrocellulose membranes. The membrane was blocked in PBS containing $5 \%$ free-fat milk for $1 \mathrm{~h}$, then incubated at $4^{\circ} \mathrm{C}$ with anti-DR4, anti-DR5, caspase-3 and $\beta$-actin antibody overnight (1:200, 1:200, 1:150 and 1:500, Santa Cruz Biotechnology, Inc. Santa Cruz, CA, USA), and then incubated with horseradish peroxidase-conjugated secondary antibody (1:2000, Pierce, Rockford, IL, USA) at $37^{\circ} \mathrm{C}$ for $1 \mathrm{~h}$. Bound antibodies were visualized by chemiluminescence reagents.

Statistical analysis. All statistical analyses were performed by SPSS12.0 (statistical package for the social science program 12.0) (SPSS Inc., Chicago, IL, USA). The data are presented as mean \pm SD and subjected to one-way ANOVA followed by Student's t-test, and $\mathrm{P}<0.05$ was considered significant.

\section{Results}

Characterization of TRAIL expression following hypoxia and irradiation. To characterize TRAIL expression following hypoxia and irradiation, TRAIL mRNA amplication products were made using DNA electrophoresis (Fig. 2A). As compared with control, TRAIL mRNA significantly increased after 4 Gy irradiation $(\mathrm{P}<0.001)$, and recombinant plasmids combined with $4 \mathrm{~Gy}$ irradiation increased TRAIL mRNA expression significantly $(\mathrm{P}<0.001)$, furthermore, after cells were transfected with recombinant plasmids, they were treated by hypoxia and irradiation, TRAIL mRNA increased the most $(\mathrm{P}<0.001)$, even compared with 4 Gy $(\mathrm{P}<0.05, \mathrm{P}<0.001)$ (Fig. 2B). In addition, secreted TRAIL concentration in culture supernatant was measured by ELISA, as compared with control group, 4 Gy irradiation alone increased protein concentration significantly $(\mathrm{P}<0.05)$, the combination with plasmids increased the protein concentration the most $(\mathrm{P}<0.001)$, while there were no obvious difference between the two plasmids. Secreted TRAIL concentration was increased the most in cells treated by recombinant plasmids transfection, hypoxia and irradiation $(\mathrm{P}<0.001)$, even compared with 4 Gy $(\mathrm{P}<0.001)$ (Fig. 2B).

Difference of radiosensitivity between transfected pc-E-hsT and pc-H/E-hsT cells under hypoxic and normoxic condition. In order to compare the difference of radiosensitivity between transfected pc-E-hsT and pc-H/E-hsT cells under hypoxic and normoxic condition, cologenic assay was introduced (21).
The survival curves were constructed and $\mathrm{D}_{0}$ value were determined. Under normoxia condition, the survival curve for cells transfected with pc-E-hsT and pc-H/E-hsT plasmids had much steeper slopes, and there was no obvious difference between two plasmids, $\mathrm{D}_{0}$ value of control, pc-E-hsT and pc-H/E-hsT group was 3.26, 1.91 and 1.89 Gy, respectively (Fig. 3A). In addition, under hypoxia condition, the survival curve for control cells, cells transfected with pc-E-hsT and pc-H/E-hsT plasmids gradually became steeper, $\mathrm{D}_{0}$ value of control, pc-E-hsT and pc-H/E-hsT group was 4.81, 2.54 and $1.13 \mathrm{~Gy}$, respectively (Fig. 3B). These results indicated that hypoxia can cause A549 cell radioresistance, pc-E-hsT can increase radiosensitivity, even in hypoxia condition, while pc-H/E-hsT can overcome radioresistance induced by hypoxia increasing radiosensitivity the most.

Transfection of cell apoptosis rates induced by hypoxia and irradiation. Cell apoptosis was attributed to the radiosensitivity of cancer cells (22), therefore, apoptotic rate was measured to identify the hypothesis. The cell apoptotic rate was subsequently calculated based on the combined percentages of early apoptosis (Annexin $\mathrm{V}^{+} / \mathrm{PI}^{-}$) and late apoptosis (Annexin $\mathrm{V}^{+} / \mathrm{PI}^{+}$) detected by flow cytometry and TUNEL (Fig. 4A). Quantification of apoptotic rate found that as compared with control group, 4 Gy irradiation could significantly induce apoptosis increase $(\mathrm{P}<0.001)$, and the roles of combination with pc-E-hsT and pc-H/E-hsT plasmid transfection, hypoxia and irradiation was more obvious $(\mathrm{P}<0.001)$, even as compared with $4 \mathrm{~Gy}(\mathrm{P}<0.05, \mathrm{P}<0.001)$, but there were no obvious difference between the plasmids. Based on transfection of plasmids, cells treated with hypoxia and irradiation, hypoxia did not increase pc-E-hsT transfected cells apoptotic rate, but promoted pc-H/E-hsT transfected cells. Additionally, apoptotic rate measured by TUNEL was similar with FCM (Fig. 4B). Taken together, plasmid carrying Egr1 promoter played inducible roles under normoxia and hypoxia, but promoter HRE only under hypoxia.

DR4, DR5 and caspase-3 expression levels after transfection, hypoxia and irradiation. TRAIL and DR4, DR5 receptors regulated apoptosis upon caspase- 8 or caspase-10 to caspase- 3 pathways, caspase- 3 cleaved fragments, then caused cell apoptosis (10). As shown (Fig. 5), 4 Gy irradiation caused DR4, DR5 and cleaved caspase- 3 increase, DR4 had not obvious change 

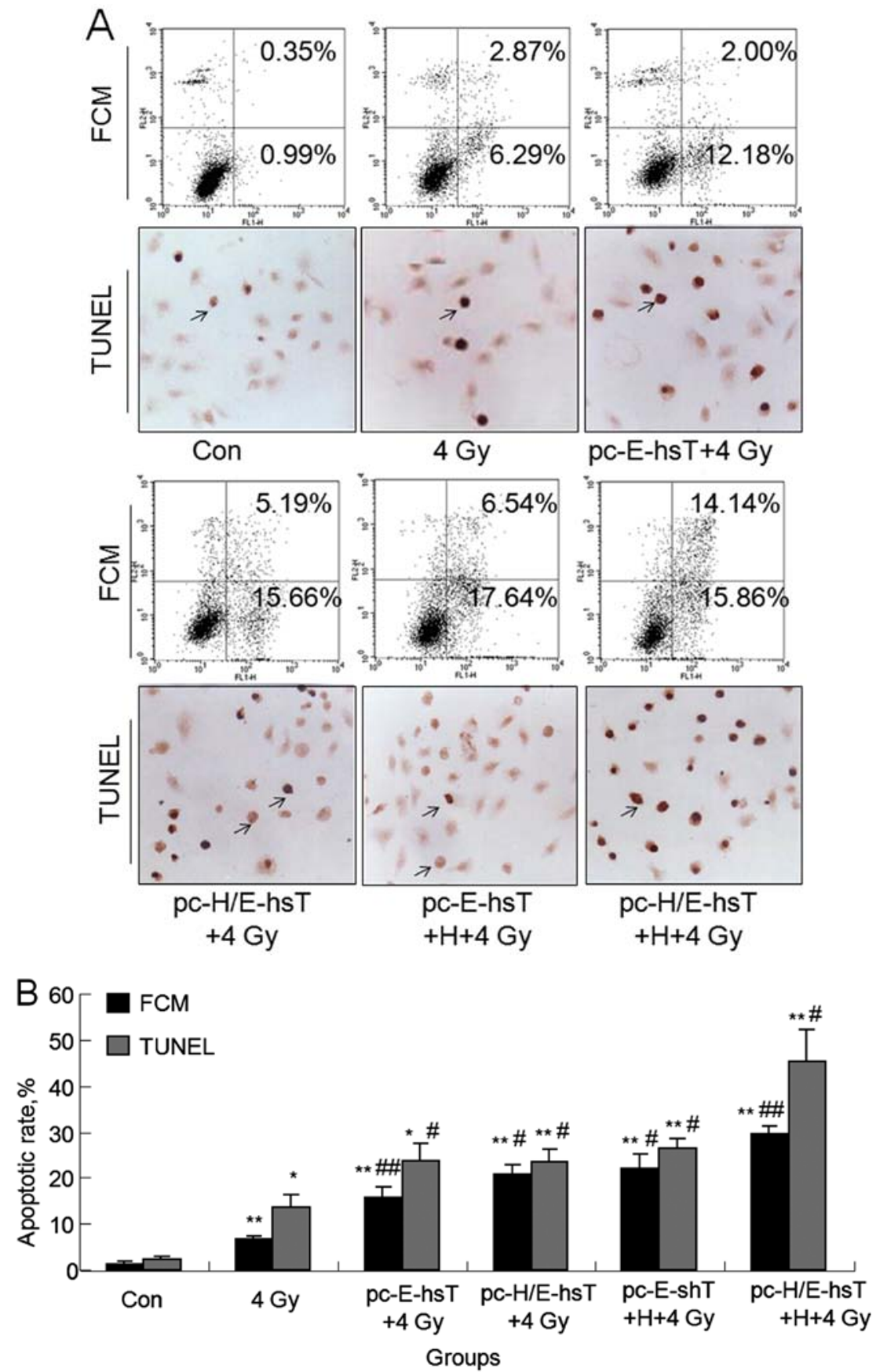

Figure 4. Apoptosis in transfected A549 cells induced by hypoxia and irradiation. (A) representative examples of apoptosis measured by FCM and TUNEL. Apoptotic cells are stained brown by TUNEL (Black arrow, x200). (B) Quantitative analysis of apoptotic rate detected by FCM and TUNEL. ${ }^{*}$ P $<0.05$ (TUNEL), ${ }^{* *} \mathrm{P}<0.01$ (FCM and TUNEL) as compared with control group, ${ }^{\#} \mathrm{P}<0.05$ (FCM and TUNEL), ${ }^{\# \#} \mathrm{P}<0.001$ (FCM) as compared with $4 \mathrm{~Gy}$.

in pc-E-hsT and pc-H/E-hsT transfected cells under normoxic and hypoxic condition. DR5 and cleaved caspase-3 increased mostly in pc-H/E-hsT transfected cells under hypoxic condition. These results also indicated TRAIL overexpression could induce A549 cell apoptosis, and its regulation was by TRAIL and DR5 to caspase-3 pathway, but not DR4.

\section{Discussion}

Tumor gene-radiotherapy is a method put forward in recent years to treat tumors. It is used to transfer an exogenous gene into cells, which can kill tumor cells and enhance radiosensitivity. It is synergistic on gene and radiation in local radiotherapy (22). It had been reported that Egr1 was transcriptionally induced by radiation exposure, and the radiation inducible role was conferred by serum response or $\mathrm{CC}(\mathrm{A} / \mathrm{T}) 6 \mathrm{GG}$ elements in its promoter region $(12,23)$. Therefore, the expression for exogenous gene controlled by Egrl promoter could be enhanced by ionizing radiation temporally, spatially and dose-dependently (24). However, Egr1 promoter inducible activity by radiation was greatly reduced under hypoxic condition, which was caused by oxygen-free radical reduction $(16,17)$. Therefore, some strategies to enhance Egrl radiation inducible characteristics were applied. Such as an adenoviral vector carrying Egr1 promoter, more efficient transferring system that controls 


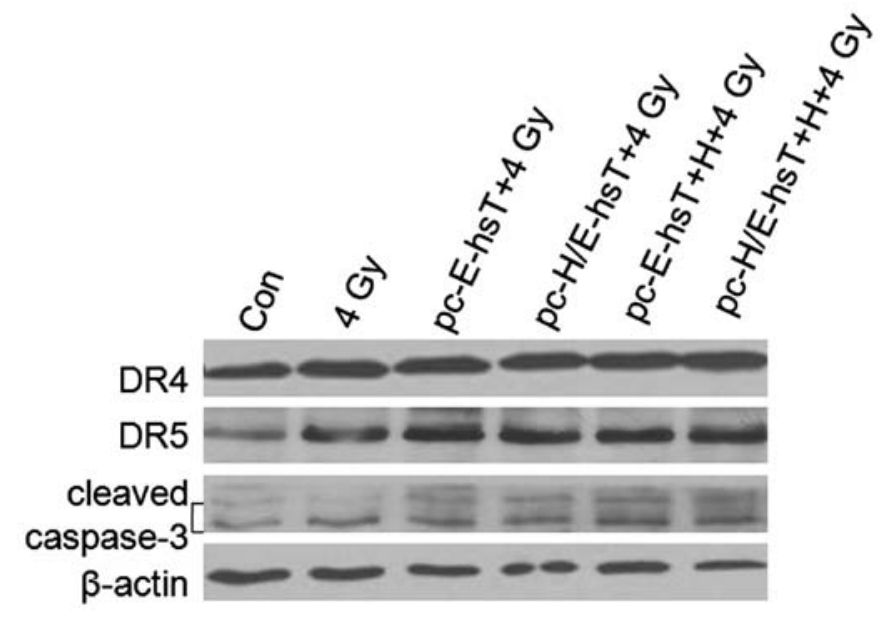

Figure 5. Expression of DR4, DR5 and cleaved caspase-3 proteins in A549 cells by western blotting. DR4 (44 kD), DR5 (43 kD), cleaved caspase-3 $(19 \mathrm{kD}$ and $17 \mathrm{kD})$ and $\beta$-actin $(42 \mathrm{kD})$ expression levels in A549 cells.

the expression of tumor necrosis factor- $\alpha$ (Ad-Egrl-TNF- $\alpha$ ) was shown to enhance anti-tumor response, TNF- $\alpha$ was preferentially activated in tumors by ionizing radiation using the system (25).

In addition, the presence or absence of molecular oxygen is known to influence the biological effect of ionizing radiation; cells obtain radioresistance under hypoxic conditions (26). Although tumor hypoxia is one of the major obstacles in radiotherapy, we can take advantage of it as a tumor-specific therapeutic target to improve tumor radiation resistance, then significantly promoting tumor killing effects. HRE/Egrl dual sensitive promoter depending on HRE inducible features under hypoxic condition could effectively improve Egrl promoter transcription ability. In the present study, we successfully constructed TRAIL gene expression system regulated by HRE/Egrl chimeric promoters.

Adverse side effects that can arise from the non-specific killing of normal cells with high doses of radiation limits the clinical application of radiotherapy (27). Consequently, strategies that sensitize tumor cells to radiation and decrease the necessary radiation dose, while increasing treatment specificity, are essential for achieving successful radiotherapy. Tumor necrosis factor-related apoptosis inducing ligand (TRAIL) was found coloned from human lymphocytes and cardiac muscle cDNA by Wiley et al, it is known as apoptosis-2 ligand (Apo-2L), and is a member of TNF superfamily (4). In recent years, by virtue of its pro-apoptotic effect on cancer cells, but not normal cells, TRAIL has been considered a promising candidate for tumor genetherapy, even tumor gene radiotherapy (5-8). Targeting TRAIL therapy, either alone or in conjunction with other therapies, has been extensively used for tumor treatment. In comparison with the soluble recombinant TRAIL protein, TRAIL gene therapy may overcome protein instability and resistance, and neighboring cancer cells untransfected by TRAIL can be killed via bystander effect $(28,29)$. In the present study, human secreted TRAIL (hsTRAIL) was mediated by HRE and Egrl together, mRNA and ELISA results showed that radiation alone can not induce increase of TRAIL expression, inducible activity of Egrl has no obvious difference under normoxic and hypoxic conditions.
TRAIL expression may increase under hypoxic condition with additional regulation of HRE, indicated that HRE/Egr1 chimeric promoters can improve the inefficient Egrl under hypoxic condition.

Cell viability, proliferation and apoptosis rates are all factors attributed to radiosensitivity of cancer cells (30). Therefore, we investigated whether overexpression of TRAIL can sensitize lung cancer cells to radiation using a clonogenic assay and cell apoptosis by FCM (31). Under normoxic condition, sensitization ability of pc-E-hsT and pc-H/E-hsT plasmids on A549 cells was basically similar, but under hypoxic condition, sensitization ability of pc-H/E-hsT plasmids was stronger than that of pc-E-hsT. Furthermore, taking into account the role of apoptosis on radiosensitivity, changes induced by radiation was analyzed, and FCM and TUNEL results showed that radiation induced cell apoptosis, TRAIL overexpression mediated by Egrl promoter induced more apoptosis. Moreover, if TRAIL overexpression was mediated by Egrl and HRE together, radiation-induced apoptosis occurred mostly under hypoxic condition. On the contrary, hypoxia was beneficial for cancer radiotherapy. In addition, TRAIL as well as DR4 and DR5 receptors regulated apoptosis upon caspase- 8 or caspase- 10 to caspase- 3 pathways, caspase- 3 was cleaved into active fragments, then caused cell apoptosis (10). In the present study, DR5 receptor played proapoptosis role with TRAIL, and DR4 receptor pro-apoptosis role was similar. Subsequently, caspase-3 was cleaved into active fragments, this also indicated apoptotic characteristics as described above.

Collectively, TRAIL overexpression co-regulated by Egr1 and HRE promoters may enhance A549 cell radiosensitivity under hypoxic condition, which have important implication for clinical radiation treatment of lung cancer. Moreover, radiosensitivity enhancement of A549 cells is related to TRAIL-mediated apoptosis depending on TRAIL and DR5 to caspase- 3 pathways. Thus, our results and conclusions provide theoretical and experimental bases for future clinical application of hypoxic lung cancer radiotherapy.

\section{Acknowledgements}

This project was supported by the National Natural Science Foundation of China (no. 81372929), Young Scholars Program of Norman Bethune Health Science Center of Jilin University (2013202017) and Basic Research and Operating Expenses of Jilin University (200903116). The pshuttle-hsTRAIL and pMD19T-Egr1 plasmids were kindly given by Dr Yan-bo Li, School of Public Health and Family Medicine, Capital Medical University.

\section{References}

1. Grade M, Wolff HA, Gaedcke J and Ghadimi BM: The molecular basis of chemoradiosensitivity in rectal cancer: Implications for personalized therapies. Langenbecks Arch Surg 397: 543-555, 2012.

2. Vogelstein B and Kinzler KW: Cancer genes and the pathways they control. Nat Med 10: 789-799, 2004.

3. Woynarowska BA, Roberts K, Woynarowski JM, MacDonald JR and Herman TS: Targeting apoptosis by hydroxymethylacylfulvene in combination with gamma radiation in prostate tumor cells. Radiat Res 154: 429-438, 2000. 
4. Wiley SR, Schooley K, Smolak PJ, Din WS, Huang CP, Nicholl JK, Sutherland GR, Smith TD, Rauch C, Smith CA, et al: Identification and characterization of a new member of the TNF family that induces apoptosis. Immunity 3: 673-682, 1995.

5. Perlstein B, Finniss SA, Miller C, Okhrimenko H, Kazimirsky G, Cazacu S, Lee HK, Lemke N, Brodie S, Umansky F, et al: TRAIL conjugated to nanoparticles exhibits increased antitumor activities in glioma cells and glioma stem cells in vitro and in vivo. Neuro Oncol 15: 29-40, 2013.

6. Tucker-Kellogg L, Shi Y, White JK and Pervaiz S: Reactive oxygen species (ROS) and sensitization to TRAIL-induced apoptosis, in Bayesian network modelling of HeLa cell response to LY303511. Biochem Pharmacol 84: 1307-1317, 2012.

7. Niemoeller OM and Belka C: Radiotherapy and TRAIL for cancer therapy. Cancer Lett 332: 184-193, 2013.

8. Li YB, Guo CX, Wang ZC, Dong LH, Guan F, Liu Y, Wang HF, Sun ZW and Gong SL: Radiosensitization of breast cancer cells by TRAIL-endostatin-targeting gene therapy. Neoplasma 60 : 613-619, 2013

9. Mahalingam D, Szegezdi E, Keane M, de Jong S and Samali A: TRAIL receptor signalling and modulation: Are we on the right TRAIL? Cancer Treat Rev 35: 280-288, 2009.

10. Sprick MR, Rieser E, Stahl H, Grosse-Wilde A, Weigand MA and Walczak H: Caspase-10 is recruited to and activated at the native TRAIL and CD95 death-inducing signalling complexes in a FADD-dependent manner but can not functionally substitute caspase-8. EMBO J 21: 4520-4530, 2002.

11. Datta R, Rubin E, Sukhatme V, Qureshi S, Hallahan D, Weichselbaum RR and Kufe DW: Ionizing radiation activates transcription of the EGR1 gene via CArG elements. Proc Nat Acad Sci USA 89: 10149-10153, 1992.

12. Weichselbaum RR, Hallahan DE, Sukhatme VP and Kufe DW Gene therapy targeted by ionizing radiation. Int J Radiat Oncol Biol Phys 24: 565-567, 1992

13. Liu LL, Smith MJ, Sun BS, Wang GJ, Redmond HP and Wang JH: Combined IFN-gamma-endostatin gene therapy and radiotherapy attenuates primary breast tumor growth and lung metastases via enhanced CTL and NK cell activation and attenuated tumor angiogenesis in a murine model. Ann Surg Oncol 16: 1403-1411, 2009

14. Yang W and Li XY: Anti-tumor effect of pEgr-interferon$\gamma$-endostatin gene-radiotherapy in mice bearing Lewis lung carcinoma and its mechanism. Chin Med J (Engl) 118: 296-301, 2005.

15. Li ZL, Liang S, Wang ZC, Li YB, Guo CX, Fang F, Gong SL and Lin $\mathrm{CH}$ : Expression of Smac induced by the Egrl promoter enhances the radiosensitivity of breast cancer cells. Cancer Gene Ther 21: 142-149, 2014.

16. Weichselbaum RR, Kufe DW, Advani SJ and Roizman B: Molecular targeting of gene therapy and radiotherapy. Acta Oncol 40: 735-738, 2001.

17. Pines A, Bivi N, Romanello M, Damante G, Kelley MR, Adamson ED, D'Andrea P, Quadrifoglio F, Moro L and Tell G: Cross-regulation between Egr-1 and APE/Ref-1 during early response to oxidative stress in the human osteoblastic HOBIT cell line: Evidence for an autoregulatory loop. Free Radic Res 39: 269-281, 2005
18. Wang WD, Chen ZT, Li R, Li DZ, Duan YZ and Cao ZH: Enhanced efficacy of radiation-induced gene therapy in mice bearing lung adenocarcinoma xenografts using hypoxia responsive elements. Cancer Sci 96: 918-924, 2005.

19. Greco O, Joiner MC, Doleh A, Powell AD, Hillman GG and Scott SD: Hypoxia- and radiation-activated Cre/loxP 'molecular switch' vectors for gene therapy of cancer. Gene Ther 13: 206-215, 2006

20. Donadelli M, Dalla Pozza E, Scupoli MT, Costanzo C, Scarpa A and Palmieri M: Intracellular zinc increase inhibits p53(-/-) pancreatic adenocarcinoma cell growth by ROS/AIF-mediated apoptosis. Biochim Biophys Acta 1793: 273-280, 2009.

21. Pauwels B, Korst AE, de Pooter CM, Pattyn GG, Lambrechts HA, Baay MF, Lardon F and Vermorken JB: Comparison of the sulforhodamine B assay and the clonogenic assay for in vitro chemoradiation studies. Cancer Chemother Pharmacol 51: 221-226, 2003.

22. Ding M, Li R, He R, Wang X, Yi Q and Wang W: p53 activated by AND gate genetic circuit under radiation and hypoxia for targeted cancer gene therapy. Cancer Sci 106: 1163-1173, 2015.

23. Cao XM, Koski RA, Gashler A, McKiernan M, Morris CF, Gaffney R, Hay RV and Sukhatme VP: Identification and characterization of the Egr-1 gene product, a DNA-binding zinc finger protein induced by differentiation and growth signals. Mol Cell Biol 10: 1931-1939, 1990.

24. Min FL, Zhang $\mathrm{H}$ and Li WJ: Current status of tumor radiogenic therapy. World J Gastroenterol 11: 3014-3019, 2005.

25. Bickenbach KA, Veerapong J, Shao MY, Mauceri HJ, Posner MC, Kron SJ and Weichselbaum RR: Resveratrol is an effective inducer of CArG-driven TNF-alpha gene therapy. Cancer Gene Ther 15: 133-139, 2008.

26. Brown JM and Wilson WR: Exploiting tumour hypoxia in cancer treatment. Nat Rev Cancer 4: 437-447, 2004.

27. Yang TJ and Ho AY: Radiation therapy in the management of breast cancer. Surg Clin North Am 93: 455-471, 2013.

28. Seol JY, Park KH, Hwang CI, Park WY, Yoo CG, Kim YW, Han SK, Shim YS and Lee CT: Adenovirus-TRAIL can overcome TRAIL resistance and induce a bystander effect. Cancer Gene Ther 10: 540-548, 2003.

29. Hu Y, Ouyang W, Wu F, Cao CH, Wang K, Liao ZK, Zhong YH, Zhou FX, Liu SQ, Xia L, et al: Enhanced radiosensitivity of SW480 cells via TRAIL up-regulation mediated by Egr-1 promoter. Oncol Rep 22: 765-771, 2009.

30. Cheng G, Kong D, Hou X, Liang B, He M, Liang N, Ma S and Liu X: The tumor suppressor, p53, contributes to radiosensitivity of lung cancer cells by regulating autophagy and apoptosis. Cancer Biother Radiopharm 28: 153-159, 2013.

31. Pauwels B, Korst AE, de Pooter CM, Pattyn GG, Lambrechts HA, Baay MF, Lardon F and Vermorken JB: Comparison of the sulforhodamine B assay and the clonogenic assay for in vitro chemoradiation studies. Cancer Chemother Pharmacol 51: 221-226, 2003. 